\title{
Assessment of water quality using environmetric techniques at Johor River
}

\begin{abstract}
In this study, the spatial water parameters of Johor River from 2003 ï 2007 were analyzed by using environmetric techniques namely the hierarchical agglomerative cluster analysis (HACA), discriminant analysis (DA) and principle component analysis (PCA). These methods were used to determine the spatial variations and sources of pollutants in Johor River. There were 23 water parameters selected namely DO, BOD, COD, SS, pH, NH3-NL, temperature, conductivity, salinity, turbidity, DS, TS, NO3, Cl, PO4, Zn, Ca, Fe, K, Mg, Na, E-coli and coliform. The HACA has grouped the water monitoring stations along the Johor River into three clusters; (1) the stations with low pollution sources (LPS), (2) moderate pollution sources (MPS) and (3) high pollution sources (HPS). Stations are classified into three clusters based on the similarity of each variable poses towards each other. Ten water parameters were being discriminated by conducting forward stepwise DA out of the 23 water quality variables. PCA were then conducted to identify the pollutant sources from the landuse of the area. Eight principle components (PCs) were obtained with $79 \%$ variation of LPS region. Five PCs were obtained from MPS with the total variation of $84 \%$ of the MPS region. The HPS has the most number of PCs which are nine PCs with a total variation of 83 $\%$ of its region. For the MPS and HPS, the pollutants were introduced by human activities such as discharge of chemicals, industrial wastewater, agricultural activities and urban development activities.
\end{abstract}

Keyword: Environmentric; Cluster analysis; Discriminant analysis; Principal component analysis; Heavy metals 